\title{
Tattoo reaction: Case series
}

\section{Muneer Mohamed, Kindy Darilyn Anderson Sohkhlet, Aneesh Samayam}

\author{
Department of Dermatology, MVJ Medical College and Research Hospital, Bangalore, India
}

Corresponding author: Dr. Muneer Mohamed, E-mail: muneermhmd@yahoo.co.in

\begin{abstract}
Tattoo is going to be a very common practice especially among young people and we are witnessing a gradual increase of numerous potential complications to tattoo placement which are often seen by physicians, but generally unknown to the public. The most common skin reactions to tattoo include a transient acute inflammatory reaction due to trauma of the skin with needles and medical complications such as superficial and deep local infections, systemic infections, allergic contact dermatitis, photodermatitis, granulomatous and lichenoid reactions, and skin diseases localized on tattooed area (eczema, psoriasis, lichen, and morphea). In this series we present three cases of tattoo reaction.
\end{abstract}

Key words: Tattoo; Lichenoid reaction; Pseudolymphomatous reaction

\section{INTRODUCTION}

Tattoos are defined as the intentional or accidental deposit of pigment into the skin. Inadvertent use of variety of dye materials in the tattoos has led to a spectrum of histological reactions. It has tremendous religious and spiritual significance. In addition, tattooing for cosmetic purposes has become quite popular in recent times. With this increasing trend, there is also an increased risk of adverse effects. Cutaneous reactions to tattoos are uncommonly reported in literature. They are generally attributed to the metallic salts used in the preparation of the pigment. Allergic reactions to a particular pigment can manifest in several ways including allergic contact dermatitis and photoallergic dermatitis.

\section{CASE REPORTS}

\section{Case 1}

Thirty two year old male presented with raised lesions over his left hand one month ago which appeared five days after he got a tattoo which was associated with itching. Red tattoo pigment was used. He gives no history of pus exudation, fever or pain.

\section{On Examination}

A star shaped erythematous plaque with scaling over tattoo was seen. Mantoux test was negative (Fig. 1).

Biopsy was done and and the histopathological examination (Fig. 2) showed hyperkeratosis, acanthosis, hypergranulosis, and mild spongiosis in epidermis. Papillary dermis shows non specific mixed inflammatory cell infiltrate. Features were of pseudoepithelomatous hyperplasia.

A final diagnosis of Reaction to Tattoo pigment was given.

Patient was started with intralesional Triamcinolone injection which was given once in three weeks.

\section{Case 2}

Twenty seven year old female presented with a raised lesion over a six month old tattoo on her right hand which presented two months after she got a tattoo done. The tattoo was of red pigment and the lesion was associated with itching. She gave no history of fever, pain,cough or exudation of material from the lesion. 


\section{On Examination}

A raised erythematous plaque over the contour of the tattoo was present (Fig. 3). No discharge was observed. Mantoux was negative.

Biopsy was done and epidermis showed hyperkeratosis and acanthosis. Subepidermis showed round cell infiltrate with lakes of extracellular dye particles. The inflammatory cell infiltrate composed of lymphocytes and few macrophages with dye particles within. Features are suggestive of tattoo reaction.

A final diagnosis of Reaction to tattoo pigment was given.

She was started with intralesional Triamcinolone injection which was given once in three weeks.

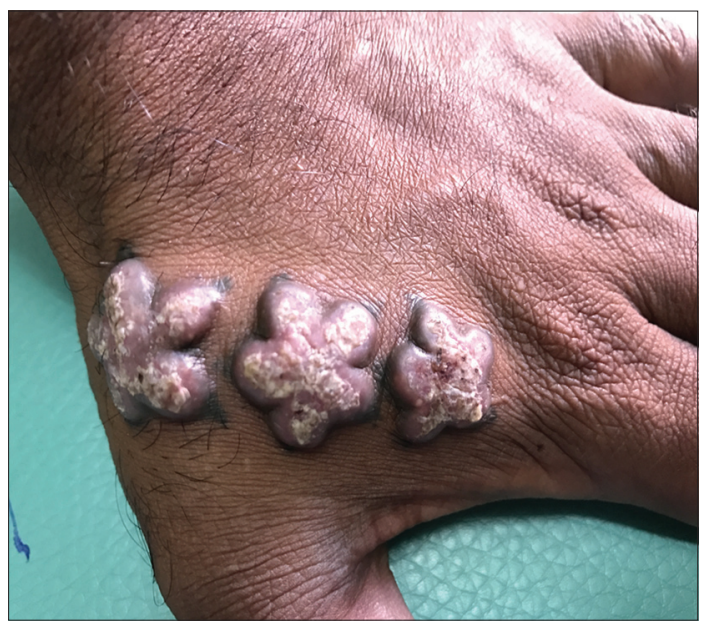

Figure 1: Star shaped erythemathous plaque with scaling over tattoo present over left hand.

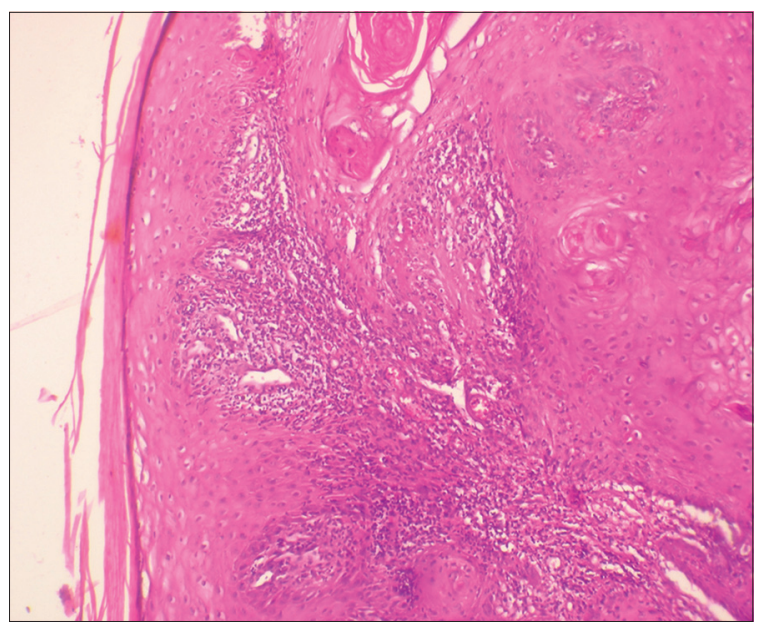

Figure 2: Epidermis- shows hyperkeratosis, acanthosis, hypergranulosis, and mild spongiosis. Papillary dermis shows non specific mixed inflammatory cell infiltrate.

\section{Case 3}

A twenty two year old male presented with redness and itching over the tattoo site since one month. Tattoo was done three month back over the right side of neck.

\section{On Examination}

A single star shaped erythematous plaque present over the right side of neck with minimal scaling was present (Figs. 4a and 4b). Mantoux was negative.

Biopsy was done and epidermis was unremarkable. Upper dermis shows dense lymphohistiocytic infiltrate around the blood vessels extending in to the deep dermis. The histiocytes contain pigment in the cytoplasm. Features are suggestive of tattoo reaction.

A final diagnosis of reaction to tattoo pigment was given.

Patient was started on intralesional triamcinolone injection which was given once in three weeks.

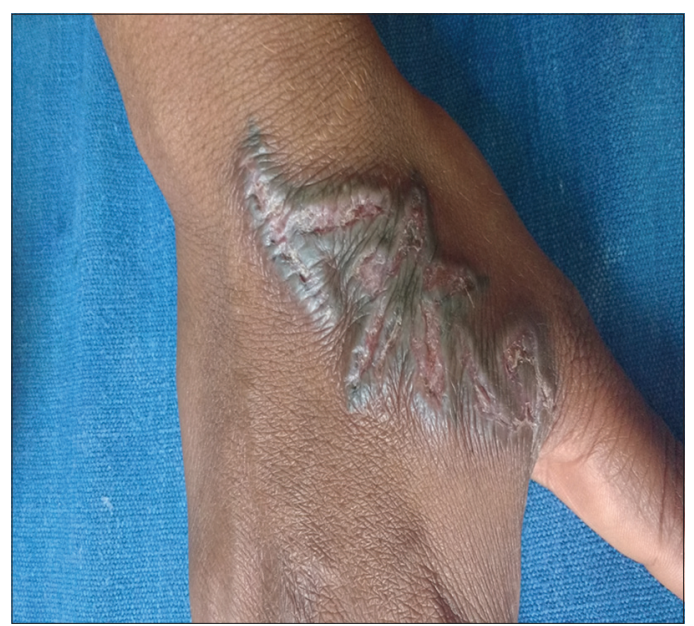

Figure 3: Raised erythematous plaques over the contour of the tattoo over right hand.

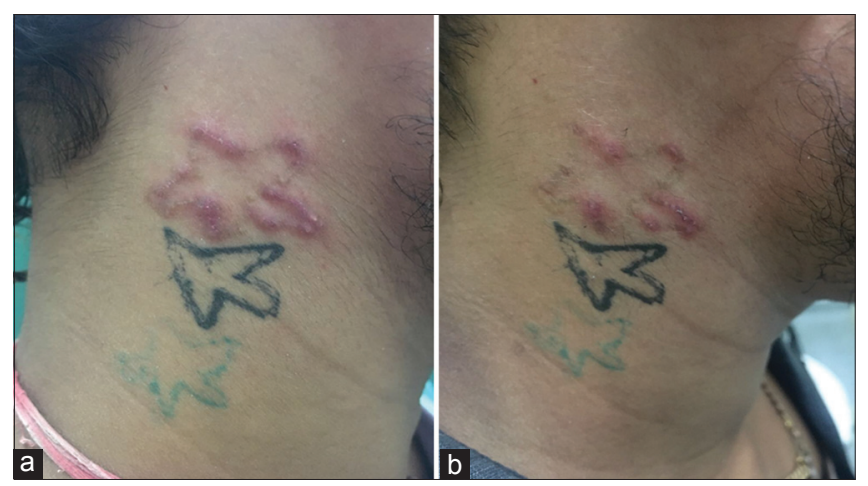

Figure 4: (a) Single star shaped erythematous plaque present over the right side of neck with minimal scaling over red colored tattoo. (b) Shows improvement after 3 sittings of Intra lesional steroid. 


\section{DISCUSSION}

Tattoos are defined as the intentional or accidental deposit of pigment into the skin [1]. Inadvertent use of variety of dye materials in the tattoos has led to a spectrum of histological reactions. They are also plagued by complications like bacterial, viral, mycotic infections, allergic disorders as well as risk of tumours like lymphoma, basal cell carcinoma, squamous cell carcinoma, keratoacanthoma among others. Tattoo colors consist of inorganic pigments, organic dyes, or a combination of both [2]. In the past, it appears that heavy metals, that were the backbone of tattooing for decades, have been replaced by organic colorants. Tattoo artists use various pigment compounds to create different colors and hues. Depending upon the compounds used and the color of the tattoo, a variety of cutaneous reactions can be expected. The composition of ink used for professional and amateur tattoo differs significantly. For amateur tattoos, carbon particles are used, while for professional tattoos, a mixture of insoluble metals with organic dyes is used. Histological reactions to tattoo ink have been described including pseudolymphomatous, lichenoid, granulomatous, scleroderma or morphealike, sarcoidal, pseudoepitheliomatous hyperplasia, allergic contact dermatitis and photoallergy. Lichenoid and sarcoidal reactions are both less common than eczematous reactions [3]. Pathogenic mechanisms implicated in reactions to tattoo pigments include a localized, T-cell mediated, delayed hypersensitivity response (lichenoid and sarcoidal reaction). In addition, allergic reactions have been observed in the form of type I and III reactions, according to Coombs and Gell classification. Cutaneous hypersensitivity reactions, although most common with red (mercuric sulphide) tattoos, have also been reported with other colors like yellow (cadmium sulphide), brown (iron oxide), blue (cobalt), purple (manganese), green (chromium), and black (carbon)tattoos.

Allergic reactions are more frequently seen to red tattoos than other colors. This is reflected in a literature review of 17 case reports by Aberer et al., which showed that red ink was responsible for 11 out of 26 reactions reported. These reactions may occur within days of the tattoo application or up to 17 years later. Lichenoid reactions are more frequently reported with red pigment tattooing that contains mercury. Clinically, verrucous papules or plaques characteristic of hyperkeratotic lichen planus are usually seen. Currently data is lacking regarding the safety of tattoo pigment ingredients. Also, none of the tattoo ink or additives are FDA approved [4].

Steroids, laser therapy, and excision are the backbone of treatment for allergic reactions to tattoos. Other methods are mechanical dermabrasion, cryosurgery and application of caustic chemicals.

Various lasers used are Argon, carbon dioxide and Q switched lasers.

\section{CONCLUSION}

These cases are being reported owing to various reactions occurring to tattoo and fewer availability of study on the same.

\section{REFERENCES}

1. Shinohara MM, Nguyen J, Gardner J, Rosenbach M, Elenitsas R. The histopathologic spectrum of decorative tattoo complications. J Cutan Pathol. 2012;39:1110-8.

2. Kulkarni VG, Patil NR, Priya C. Tattoo pigment reactions- A case series. Indian J Basic App Med Res. 2015;4;190-4.

3. Jacks S, Zirwas M, Mossner J. A case of generalized lichenoid tattoo reaction. J Clin Aesth Dermatol. 2014;7;48-50.

4. Sanghavi SA, Dongre AM, Khopkar US. Tattoo reactions-An epidemic on the surge: A report of 3 cases. Indian J Dermatol Venereol Leprol. 2013;79;231-4.

Copyright by Muneer Mohamed, et al. This is an open-access article distributed under the terms of the Creative Commons Attribution License, which permits unrestricted use, distribution, and reproduction in any

medium, provided the original author and source are credited.

Source of Support: Nil, Conflict of Interest: None declared. 